\title{
Non-invasive continuous positive airway pressure in monolateral lung transplant patient with pneumonia and IPF
}

\author{
S. Aliberti1, M.C. Andrisani2, P. Tarsia1, R. Trevisan2, R. Cosentini3, \\ L. Longhi², M. Pappalettera1, M. Nosotti1 ${ }^{1}$, F. Blasi ${ }^{1}$
}

\begin{abstract}
Non-invasive continuous positive airway pressure in monolateral lung transplant patient with pneumonia and IPF. S. Aliberti, M.C. Andrisani, P. Tarsia, $R$. Trevisan, R. Cosentini, L. Longhi, M. Pappalettera, M. Nosotti, F. Blasi.

Patients who undergo lung transplantation are prone to develop lower respiratory tract infections, leading to severe acute respiratory failure (ARF). Endotracheal intubation may not be indicated in these patients in light of a higher rate of mortality due to infections. The application of non-invasive ventilation could play a role in bridging
\end{abstract}

\begin{abstract}
these patients through the episode of ARF waiting for medical treatment to have effect. We report the evidence of morphological and physiological effects of the application of non-invasive continuous positive airway pressure during ARF sustained by pneumonia in a patient who underwent left lung transplantation because of idiopathic pulmonary fibrosis (IPF). We studied the effects of the application of positive end-expiratory pressure on both the right native lung affected by IPF and the transplanted lung affected by pneumonia.

Monaldi Arch Chest Dis 2010; 73: 4, 169-175.
\end{abstract}

Keywords: CPAP, Pneumonia, Idiopathic pulmonary fibrosis, CT, Echo.

1 Dipartimento toraco-polmonare e cardio-circolatorio, University of Milan, IRCCS Fondazione Po.Ma.Re, Milan,

2 Radiology Department, IRCCS Fondazione Po.Ma.Re, Milan,

3 Emergency Medicine Department, IRCCS Fondazione Po.Ma.Re, Milan, Italy.

Correspondence: Stefano Aliberti MD, Dipartimento toraco-polmonare e cardio-circolatorio, University of Milan, IRCCS Fondazione Po.Ma.Re, Via Francesco Sforza 35, Milan, Italy; e-mail: stefano.aliberti@ policlinico.mi.it

A 67 yr-old Caucasian man was referred to our Respiratory Diseases Department because of a two-day history of fever and shortness of breath. Eight months before, he underwent left lung transplantation because of end-stage idiopathic pulmonary fibrosis. On admission, the patient was tachypnoic with use of the accessory muscles. Physical examination showed arterial blood pressure of $120 / 80 \mathrm{mmHg}$, heart rate of $98 \mathrm{bpm}$, temperature of $37.6^{\circ} \mathrm{C}$, respiratory rate of $40 /$ minute with an oxygen saturation of 0.95 on reservoir mask with $15 \mathrm{~L} / \mathrm{m}$ of oxygen. On chest auscultation, crackles in the middle-inferior part of the left hemithorax were present, while decreased breathing sounds were detected on the right hemithorax. Cardiovascular, abdominal and neurological examination were unremarkable. Leukocyte count was 10.9 × $10^{9}$ cells.L-1 and C-Reactive protein was $4.6 \mathrm{mg} / \mathrm{dl}$ (normal vaues: 0-0.5). Serum chemistries, renal and liver function tests were in the normal range. Blood cultures were negative. Hypoxemic acute respiratory failure was diagnosed on blood gas evaluation with $\mathrm{pH} 7.46$, Pa$\mathrm{CO}_{2} 36 \mathrm{mmHg}, \mathrm{HCO}_{3}-26$ and $\mathrm{PaO}_{2} / \mathrm{FiO}_{2}$ ratio 165. A chest computed tomography (CT) on admission showed diffuse peribronchial ground- glass opacities and consolidative areas involving the left transplanted lung, particularly in the lower lobe, see figure 1 . The right native lung showed a typical radiological pattern of IPF characterized by irreversible fibrosis and basal honeycombing. Em-

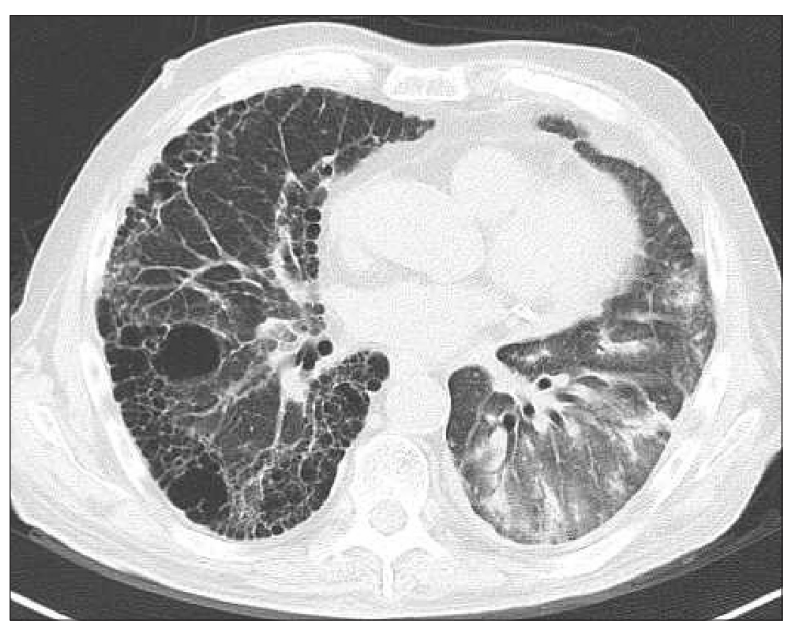

Fig. 1. - Chest computed tomography on admission showing diffuse peribronchial ground-glass opacities and consolidative areas in the left transplanted lung, and an idiopathic pulmonary fibrosis pattern in the right native lung. 
piric antibiotic therapy with ceftazidime, levofloxacin and voriconazol was commenced.

Despite high flow oxygen therapy with Venturi Mask with an $\mathrm{FiO}_{2}$ of 0.5 , the patient remained dyspnoeic and tachypnoic during the first four days of hospitalisation. On day five, in view of the high oxygen dependence and the persistence of hypoxemic acute respiratory failure $\left(\mathrm{PaO}_{2} / \mathrm{FiO}_{2}\right.$ ratio: 150), he underwent a trial of non-invasive continuous positive airway pressure (CPAP). CPAP was delivered through a high-flow generator (VitalSigns inc., Totowa, USA; 90-140 L/min) using a helmet (StarMed, Italy) as interface with a positive end-expiratory pressure (PEEP) valve (VitalSigns, USA) of $10 \mathrm{cmH}_{2} \mathrm{O}$ and a $\mathrm{FiO}_{2}$ of 0.50 .

During CPAP treatment, the patient experienced a reduction in the shortness of breath and respiratory rate (down to 20/minute) with an improvement of gas exchange $\left(\mathrm{PaO}_{2} / \mathrm{FiO}_{2}\right.$ ratio up to 312). At that time, a chest CT, a lung sonography, as well as an transthoracic echocardiography (TTE) were performed with the patient breathing on Venturi mask and, immediately after, during CPAP treatment. This case does not meet criteria for research and formal review was waived by the IRB of the IRCCS Fondazione Po.Ma.Re, Milan, Italy.

Two volumetric CT examinations were performed with a 64-slice CT scanner (Somaton Definition 64, Siemens, Germany) without intravascular contrast material with the patient lying in supine position, during a full inspiration. The same protocol was applied with the patient breathing on Venturi mask and during the application of CPAP. Lung abnormalities were evaluated with a visual score and with the use of Siemens Pulmo CT Software [1-2], in order: 1) to detect and outline lung margins; 2) to calculate lung cross-sectional areas and pixel-attenuation statistics of individual image sections; 3 ) to calculate lung volumes using different density ranges: right lung 1024 Hounsfiled Unit (HU) to $-200 \mathrm{HU}$; left lung $-1024 \mathrm{HU}$ to $+100 \mathrm{HU}$; 4) to show graphic display of results. The following findings were evaluated on both lungs: 1) lung height and total lung volume; 2) lung density expressed as mean of $\mathrm{HU}$ was evaluated for the whole lung and at different levels; 3) histograms of the attenuation-frequency distributions, plot of attenuation (HU) versus the frequency distribution of pixels $(\%)$ of the basal section [3]; 4) the Full Width at Half Maximum (FWHM), representing the difference between two extreme density values and expressed in HU, at which the relative frequency of pixels, expressed in percentage, is equal to half of its maximum value [4]. Furthermore, the following findings were evaluated on the left lung: 1) attenuation of consolidations during CPAP, by the density masks method with a subrange of density of $500 \mathrm{HU}$ to $+100 \mathrm{HU}$, expressed in percentage of red pixels; 2) expansion gradient from gravity-dependent to nondependent regions by segmentation of the basal lung scan in 5 segments of the same height. The following findings were evaluated on the right lung: 1) a semi-quantitative analysis of the IPF pattern based on the Warrick visual score $[5]$; 2) the volume changing of two different emphsematous bullas: one surrounded of more than $50 \%$ of normoaerated lung and one honeycombing bulla.

The 3D reconstructions of the chest CT scans of both lungs with the patient breathing on Venturi mask and during CPAP treatment are depicted in figure 2. A similar increase in lung volume was detected for both left and right lung during CPAP treatment, in comparison to Venturi mask, as demonstrated by the chest CT quantitative analysis presented in table 1 .

Left lung. A decrease in density of the whole left lung was detected during CPAP treatment (mean \pm SD: $-618 \pm 215 \mathrm{HU}$ ) in comparison to Venturi mask (mean \pm SD: $-563 \pm 250 \mathrm{HU}$ ). This finding was confirmed by the evaluation of single CT scans at the apex, mid region and base. A further analysis of lung density, including histograms, was performed at the level of the right inferior pul-

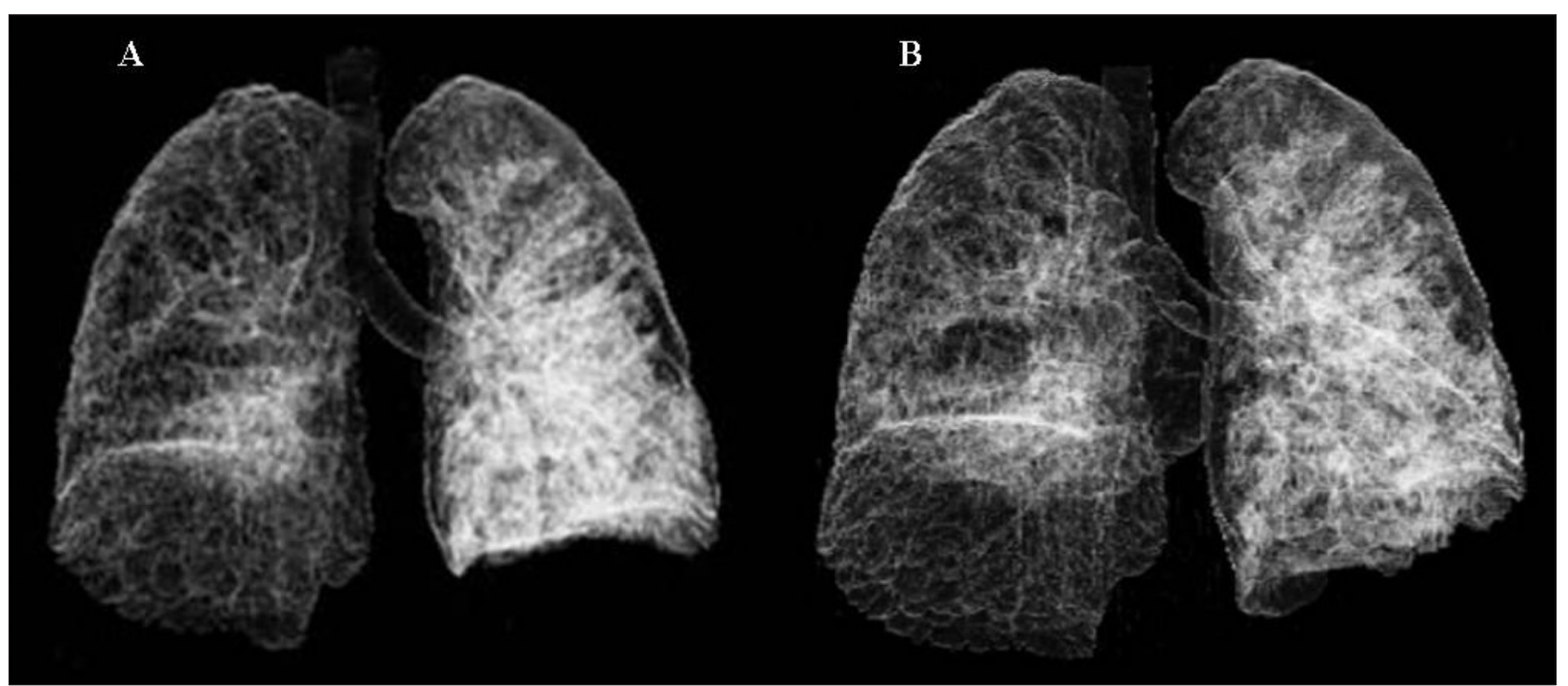

Fig. 2. - Rendered 3D reconstruction in antero-posterior view of the right (idiopathic pulmonary fibrosis) and left (transplanted with pneumonia) lung with the patient breathing on Venturi mask (A) and during CPAP treatment (B). 
Table 1. - Chest CT quantitative measures of both lungs with the patient breathing on Venturi mask and during CPAP treatment

\begin{tabular}{lcccc}
\hline & \multicolumn{2}{c}{ Right Lung } & \multicolumn{2}{c}{ Left Lung } \\
\hline & Venturi Mask & CPAP & Venturi Mask & CPAP \\
\hline Volume, $\mathrm{cm}^{3}$ & 1940 & 2310 & 1705 & 2014 \\
Height, $\mathrm{cm}$ & 19.5 & 26.2 & 19.7 & 24.8 \\
Evaluation ranges & $-1024 \mathrm{HU}$ to $-200 \mathrm{HU}$ & $-1024 \mathrm{HU}$ to $+100 \mathrm{HU}$ \\
\hline
\end{tabular}

CPAP: continuous positive airway pressure.

monary vein, see figure 3 . Histograms showed a decrease in lung density during CPAP treatment (mean \pm SD: $-534 \pm 224 \mathrm{HU}$ ) in comparison to Venturi mask (mean \pm SD: $-427 \pm 249$ HU) and a distribution of the curve shifted to the left with an increase of its peak. Moreover, a reduction of the FWHM during CPAP treatment (248 HU) was detected in comparison to Venturi mask (305 HU).

Density masks obtained at the basal level showed a reduction of the percentage of red pixel, representing the consolidative areas, during CPAP treatment in comparison to Venturi mask, see figure 4. Segmentation of the basal scan of the left lung in five slices of same heights showed a changing in lung density in both gravity-dependent and non-dependent regions during CPAP treatment in comparison to Venturi mask, see figure 5.

Right lung. The evaluation of the whole right lung with IPF showed no considerable changes in lung density during CPAP treatment (mean \pm SD: $797 \pm 153 \mathrm{HU}$ ) in comparison to Venturi mask (mean \pm SD: $-774 \pm 157$ HU). The effect of CPAP on the IPF lung was evaluated on CT scans detected at two levels: 1) at the carina, where both normal lung and ground glass areas were present, and 2) at the base, right inferior pulmonary vein, characterised by the presence of honeycombing, see figure 6 . At the carina, a small reduction of lung density was detected during CPAP treatment with a FWHM value decreasing from 115 to $98 \mathrm{HU}$. A bulla that was surrounded for more than $50 \%$ by a normoaerated lung showed a volume of $9.3 \mathrm{~cm}^{3}$ with the patient breathing on Venturi mask increasing up to $10.3 \mathrm{~cm}^{3}$ during CPAP treatment. At the base, a minimal reduction in lung density was detected during CPAP treatment. Histograms obtained at this level showed no shift to the left of the distribution of the curve, with no change in FWHM between Venturi mask (202 HU) and CPAP treatment (199 HU), demonstrating no increase of negative density values (air-space peak). A bulla in the honeycombing contest showed a volume of $3.8 \mathrm{~cm}^{3}$ with the patient breathing on Venturi mask and 4.8. $\mathrm{cm}^{3}$ during CPAP treatment.

Ground-glass opacities, as expression of alveolitis, were localised at the anterior segment of the upper lobe, at the medial segment of medial lobe and at the posterior-basal segment of the lower lobe. The alveolitis index score obtained with the patient breathing on Venturi mask was 2 (score ranges 0-4). Irregularities in the pleural margins, septal and subpleural lines, honeycombing and

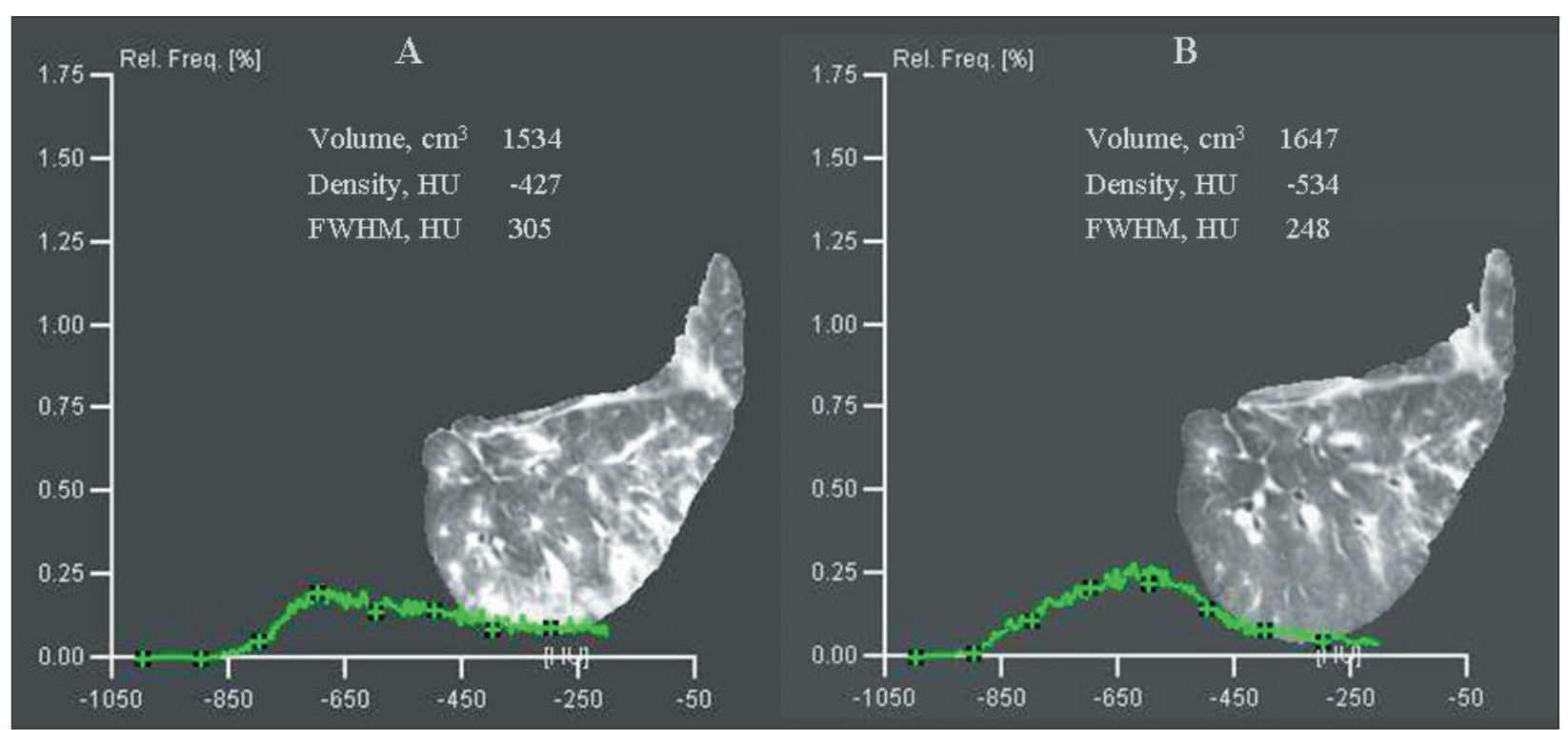

Fig. 3. - CT scan of the left lung (transplanted with pneumonia) at the right inferior pulmonary vein level (base) with attenuation-frequency distributions displayed as histograms on Venturi mask (A) and during CPAP treatment (B). On the $x$ axis the attenuation distribution from air to water, on the $y$ axis the relative frequency of pixels. 




Fig. 4. - Density mask the left lung (transplanted with pneumonia) at the right inferior pulmonary vein level (base) that highlights pixels between -500 and $+100 \mathrm{HU}$ on Venturi mask (A) and during CPAP treatment (B).

thin walled sub-pleuric cystic areas were detected, typically predominant in lower-lobe with peripheral distribution, resulting in a fibrosis index score of 23 (score ranges 0-26). Thus, the total semi-quantitative evaluation based on the Warrick visual score of the right lung with the patient breathing in Venturi mask was 25 (range 0-30). During CPAP treatment, no changes in the Warrick visual score were detected.
A convex 3.5- to 5-MHz probe (Megas CVX, Esaote Medical Systems, Florence, Italy) was used to perform chest ultrasound (US) (Vivid 3; GE Medical Systems) at the bedside with the patient in sitting position. Both lungs were studied with probe set parallel to the ribs along the $8^{\text {th }}$ intercostal space on the scapular line and with the patient breathing in Venturi mask and during CPAP treatment.

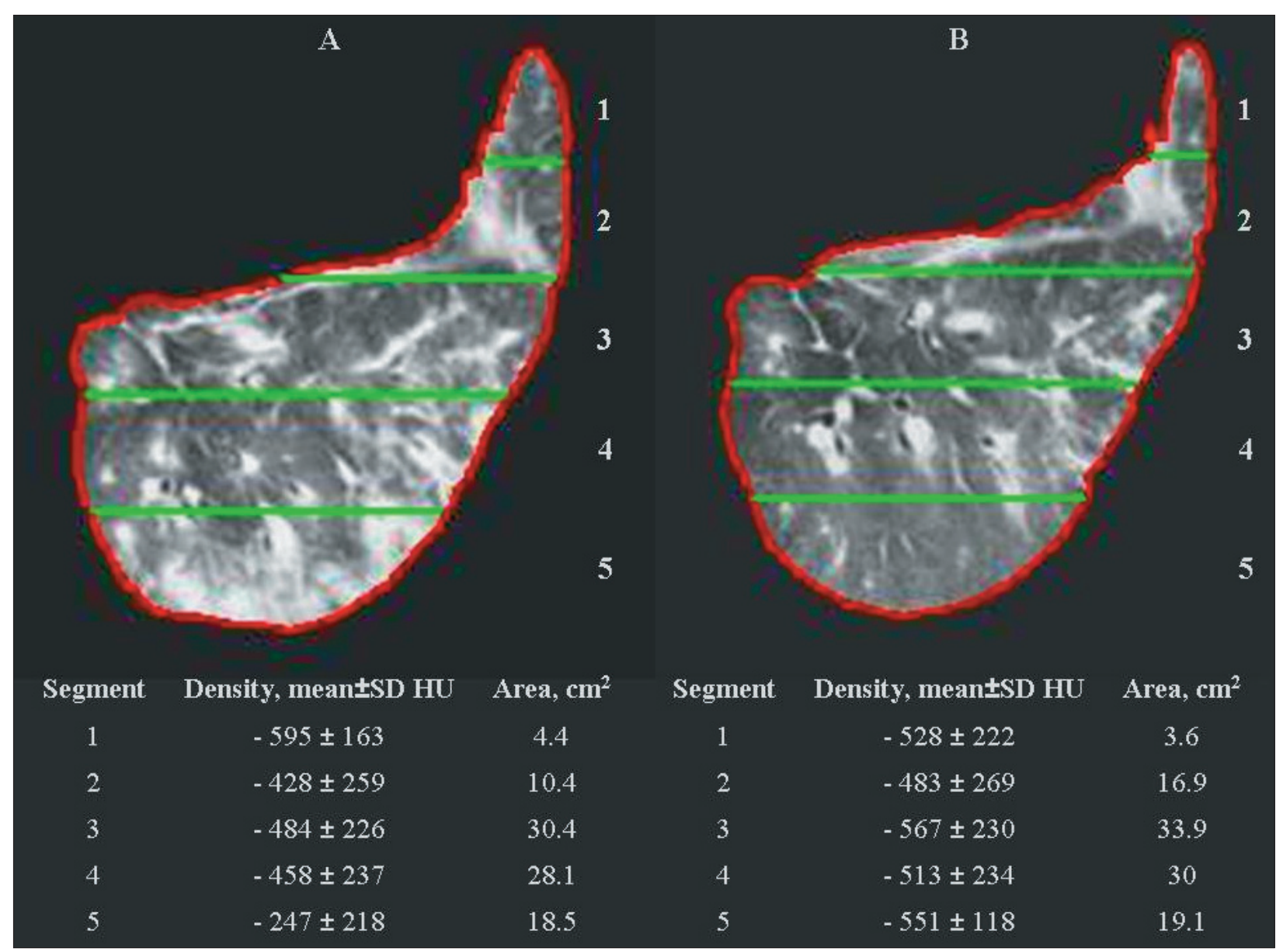

Fig. 5. - CT scan of the left lung (transplanted with pneumonia) at the right inferior pulmonary vein level (base) on Venturi mask (A) and during CPAP treatment (B), in regard to five different segments. 


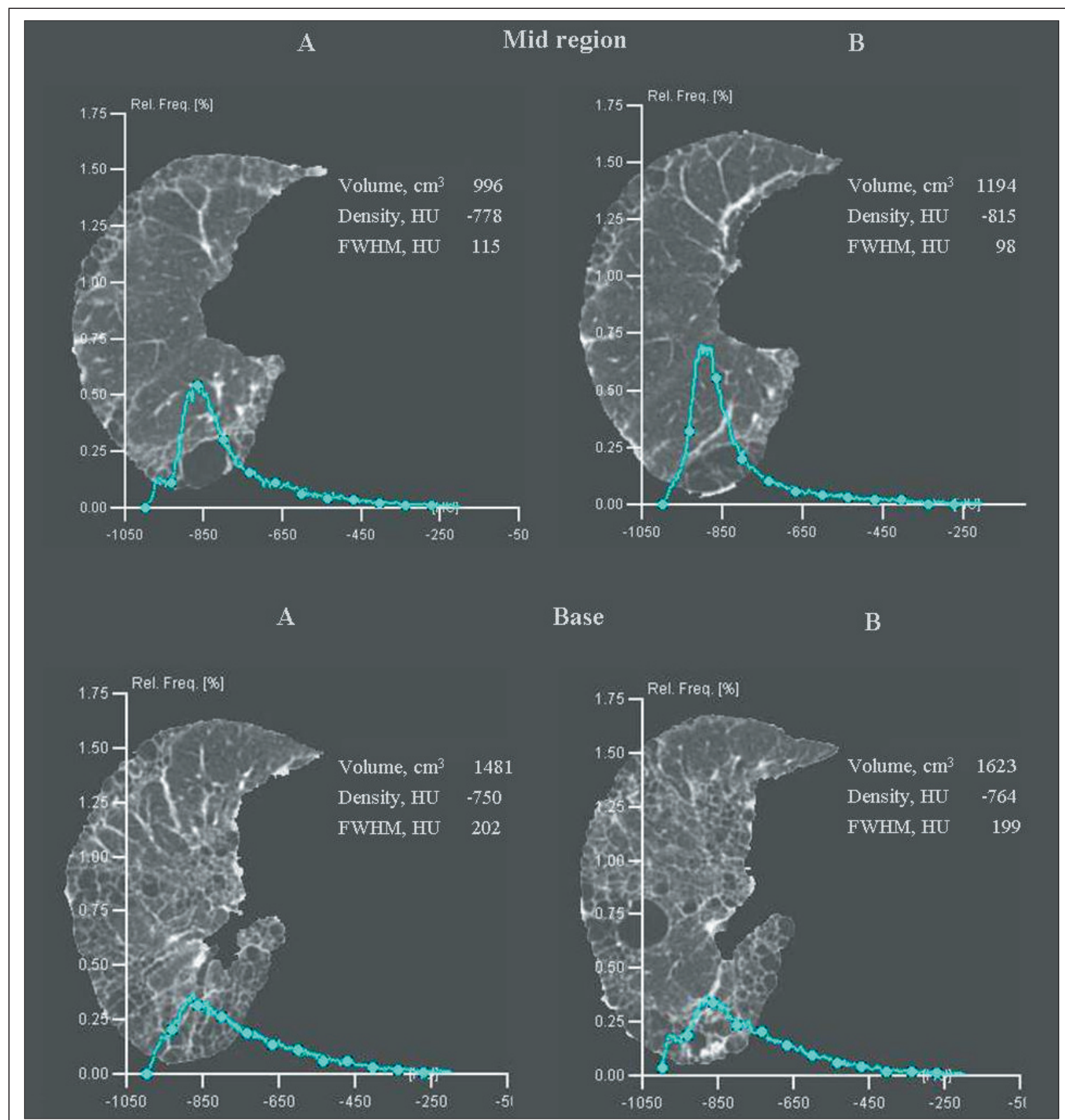

Fig. 6. - CT scan of the right lung (idiopathic pulmonary fibrosis) at the level of the carina (mid region) and right inferior pulmonary vein level (base) with attenuation-frequency distributions displayed as histograms on Venturi mask (A) and during CPAP treatment (B). On the $x$ axis the attenuation distribution from air to water, on the $y$ axis the relative frequency of pixels.

Chest US pattern of the left lung is shown in figure 7. Pleura was detected as an echogenic line with a continuous intrinsic movement during breathing (the "lung sliding sign"). With patient breathing on Venturi mask, vertically oriented "comet-tail" artefacts (B lines) were detected in the left lung, raising from pleural-lung interface up to the edge of the screen and moving with lung. B lines result from the fluid-rich subpleural interlobular septae surrounded by air and indicate the presence of an alveolar-interstitial syndrome. During the application of CPAP, a reduction of B lines in the left lung was detected. The chest US appearance of the right lung with the patient breathing on Venturi mask was also characterised by an alveo- lar-interstitial syndrome. This finding did not change during the application of CPAP.

Echocardiography measurements were performed using a Vivid 3, GE Medical Systems, using standard windows and in agreement with the current guidelines suggested by the American Society of Echocardiography [6]. A reduction of the TD and TS volumes of the left ventricle was detected during CPAP treatment $(74$ and $25 \mathrm{~mL}$, respectively), in comparison to Venturi mask (101 and $37 \mathrm{~mL}$, respectively). An increase in the ejection fraction, from 0.63 to 0.66 was detected during CPAP treatment. The diastolic evaluation showed a reduction of the E/A ratio during CPAP treatment (0.42) in comparison to Venturi mask (0.72). 


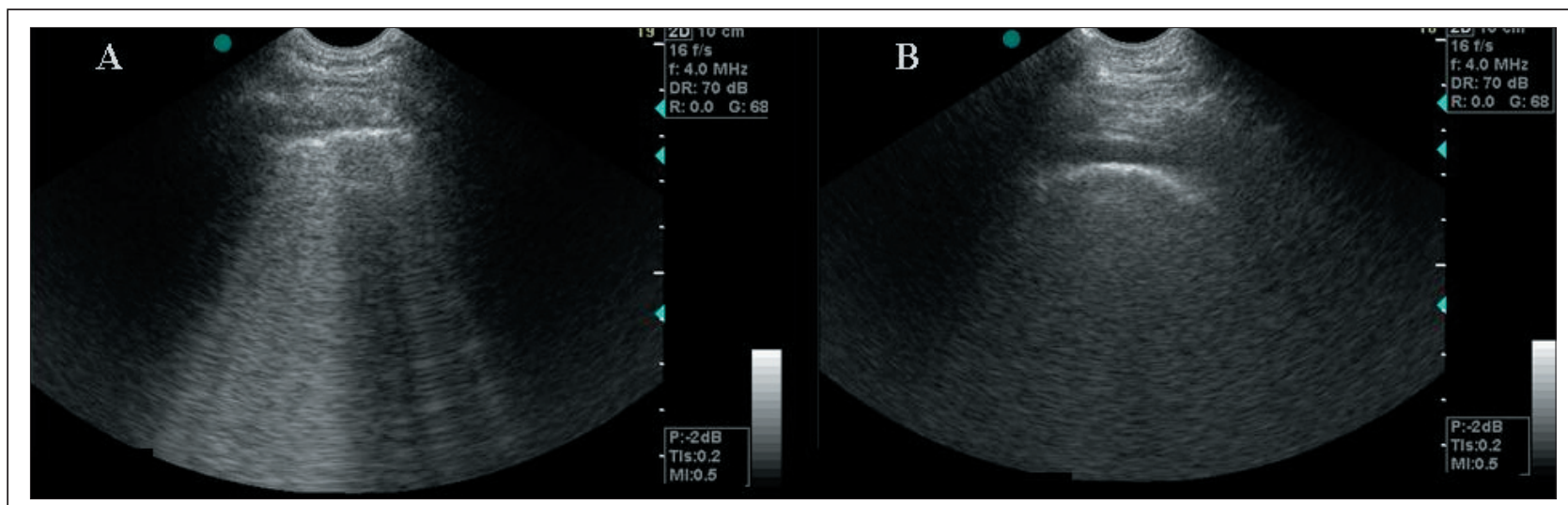

Fig. 7. - Chest US pattern of the left lung (transplanted with pneumonia) on Venturi mask (A) and during CPAP treatment (B).

\section{Discussion}

To our knowledge, this is the first case report showing the morphological evidence of the effect of non-invasive CPAP on both pneumonia and IPF. We found that the application of CPAP equally increased lung volumes of two hemithoraces affected by different diseases, IPF and pneumonia. The application of PEEP on the IPF lung resulted in minimal overdistention, while in the transplanted lung with pneumonia we detected a recruitment of consolidative areas.

Regarding the effect of PEEP on the fibrotic lung, in addition to the overall overdistension, the size of the bulla was found to increase, particularly in the honeycomb area. The potential detrimental effects include increased work of breathing and increased risk of pneumothorax. Any negative effect on lung mechanics was offset by the beneficial recruitment of the left lung, as testified by the decrease in respiratory rate and improvement in gas exchange. We are unable to quantify the potential increased risk of pneumothorax due to increased bull size.

In the transplanted lung with pneumonia, the recruitment of consolidative and atelectatic areas was demonstrated both by CT scan and lung sonography. It is well known that a CT scan is capable of differentiating between recruitment (aeration of previously gasless lung regions) and inflation (further aeration of already inflated lung regions). This technique has been previously used to evaluate PEEP in preventing end-expiratory collapse and lung recruitability in patients with acute lung injury and ARDS [7]. During an episode of pneumonia, the increased lung mass and superimposed pressure squeeze out gas from the most dependent, and, to a lesser extent, non dependent regions, leading to the $\mathrm{CT}$ appearance of gasless regions. In our patient, the application of PEEP contributed to persistent patency of lung regions that would otherwise have collapsed at end-expiration. This lead to recruitment of consolidative areas and reduction of lung density. The regional lung analysis also confirmed the effect of PEEP in both dependent and nondependent portions of the lung.

Lung ultrasonography confirmed the recruitment effect of CPAP on consolidative/atelectasis areas in the transplanted with pneumonia. This finding was sustained by the reduction of the interstitial syndrome, B lines, during the application of a PEEP in comparison to Venturi mask. To our knowledge, this is the first report in the literature regarding the utility of chest US in the evaluation of the recruitment effect of non-invasive CPAP in a patient with pneumonia. Compared to CT scanning, chest US has undoubted advantages in terms of reproducibility, ready availability, and lack of radiation exposure. Further studies would therefore be needed to confirm the possible role of this technique in defining the recruitment of consolidative areas in patients with pneumonia undergoing CPAP and in optimising the level of pressure to use. After its complete evaluation in prospective clinical trials, this technique could be applied in transplant patients with respiratory infections as a "bridge" in sustaining the respiratory system during the first days of antibiotic therapy. In our case report, it took four days to start noninvasive CPAP because of possible contraindications of this technique in our patient, mainly pneumothorax. However, after a deeper analysis of the risk-benefit ratio of applying pressures into our patient's lungs, we decided to perform a trial with a helmet CPAP. This decision was supported by the fact that this trial was performed in a respiratory intensive care unit of a large hospital with a rapid access to the ICU for early airway protection in case of a lack of improvement.

\section{References}

1. Gierada DS, Yusen RD, Pilgram TK, et al. Repeatability of quantitative CT index of emphysema in patients evaluated for lung volume reduction surgery. Radiology 2001; 220: 448-454.

2. Sverzellati N, Calabrò E, Chetta A, et al. Visual score and quantitative $\mathrm{CT}$ indices in pulmonary fibrosis: relationship with physiologic impairment. Radiol Med 2007; 112: 1160-1172.

3. Palumbo A, Luccichenti G, Belgrano M, et al. Threedimensional quantitative assessment of lung parenchima in cystic fibrosis: preliminary results. Radiol Med 2007; 112: 21-30.

4. Best AC, Meng J, Lynch AM, et al. Idiopathic pulmonary fibrosis: physiologic tests, quantitative CT indexes, and CT visual score as predictors of mortality. Radiology 2008; 246: 935- 940. 
5. Bellia M, Cannizzaro F, Scichilone N, et al. HRCT and sclerodermia: semiquantitative evaluation of lung damage and functional abnormalities. Radiol Med 2009; 114: 190-203.

6. ASE Committee Recommendations. Recommendations for Chamber Quantification: A Report from the American Society of Echocardiography's Guidelines and Standards Committee and the Chamber Quantification
Writing Group, Developed in Conjunction with the European Association of Echocardiography, a Branch of the European Society of Cardiology. J Am Soc Echocardiogr 2005; 18: 1440-1463.

7. Caironi P, Langer T, Gattinoni L. Acute lung injury/acute respiratory distress syndrome pathophysiology: what we have learned from computed tomography scanning. Curr Opin Crit Care 2008; 14: 64-9.

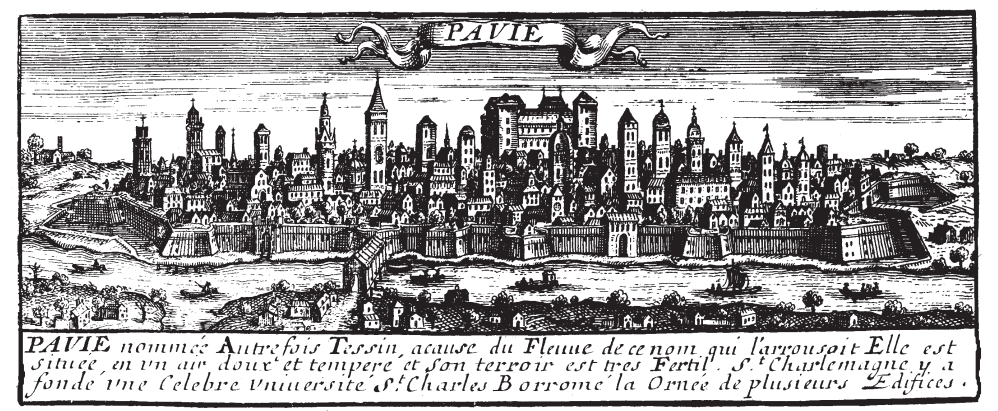

